\title{
Additional Abscissas and Weights for Gaussian Quadratures of High Order: Values for $n=64,80$, and 96
}

\author{
Philip Davis and Philip Rabinowitz ${ }^{1}$
}

Abscissas and weights for Gaussian quadratures of orders $n=64,80$, and 96 are given to twenty decimal places.

In a previous paper, ${ }^{2}$ tables of zeros of the Legendre polynomials $P_{n}(x)$ were published for various values of $n$ up to $n=48$. It was also stated there that values were available for $n=64,80$, and 96 . Despite a brisk demand for these values, they were not published with the others because doubt was cast on their accuracy. It was feared that the round-off error accumulated in using the recursion formula for Legendre polynomials

$$
\begin{aligned}
& P_{n}(x)=\left(2-\frac{1}{n}\right) x P_{n-1}(x)-\left(\frac{n-1}{n}\right) P_{n-2}(x) \\
& P_{0}(x)=1, \quad 1 P_{1}(x)=x
\end{aligned}
$$

would propagate to such an extent that for high values of $n$ a significant loss of accuracy would result.

These values are now published since we have been able to show that these fears were unfounded. The way this was accomplished was as follows: $P_{n}\left(x_{k n}+5.10^{-22}\right) \quad\left(k=1, \ldots, \frac{n}{2}\right)$ was evaluated on

\footnotetext{
1 Present address, Weizmann Institute, Rehovoth, Israel. 2 P. Davis and P. Rabinowitz, A bscissas and weights for Gaussian quadra-
of high order, J. Research NBS 56, 35 (1956) RP2645.
}

the WEIZAC (Weizmann Automatic Computer), using triple-precision operations, with the thought that if $P_{n}\left(x_{k n}+5.10^{-22}\right)$ would be of different sign than $P_{n}\left(x_{k n}-5 \cdot 10^{-22}\right)$, then $x_{k n}$ would be a correct 21-place zero of $\mathrm{P}_{n}(x)$, The only triple-precision operations needed were addition, subtraction, and multiplication. In addition, the triple-precision quotient of two single-precision numbers was needed. An interpretive routine simplified the program for evaluating $\mathrm{P}_{n}(x)$.

The results of this computation were that the SEAC (Standards Electronic Automatic Computer) values were all correct to 21 places with one exception; one value was in error by 1 in the 21st place and has been corrected in the present table.

Because the weights were computed by the formula

$$
a_{k n}=\frac{2\left(1-x_{k n}^{2}\right)}{\left[n P_{n-1}\left(x_{k n}\right)\right]^{2}} k=1,2, \ldots, n / 2,
$$

and because $P_{n}\left(x_{k n}\right)$ was found to be correct to within 1 unit in the 21 st place, it was felt that $P_{n-1}\left(x_{k n}\right)$ was also correct to 21 places and hence $a_{k n}$ can be considered correct to the 20 places given in the table.

In the tables, only the abscissas lying between 0 and 1 have been listed. 
$\mathrm{n}=64$

0.9993050417

0.9963401167

0.9910133714

0.9833362538

0.9733268277

0.9610087996

0.9464113748

0.9295691721

0.9105221370

0.8893154459

0.8659993981

0.8406292962

0.8132653151

0.7839723589

0.7528199072

0.7198818501

0.6852363130

0.6489654712

0.6111553551

0.5312794640

0.4894031457

0.4463660172

0.4022701579

0.3572201583

0.3113228719

0.2646871622

0.2174236437

0.1696444204

0.1214628192

0.0729931217

0.0243502926
35772139457 71955279347 76744320739 84625956931 89910963742 52053718919 58402816062 31939575821 78502805756 95114105853 52580362752 22797559742 43341407610 60531896612 71610826849 54233242564 54657339858 72393250249 02634034284 19894545658 07052957479 53464087985 63991603696 37668115950 90210956158 08767416374 40007084150 23992818037 96120554470 87799039450 63424432509 54092819761

$\mathrm{n}=80$

0.9995538226 0.9976498643 0.9942275409

0.9892913024

0.9828485727

0.9749091405

0.9654850890

0.9545907663

0.9422427613

0.9284598771

0.9132631025

0.8966755794

0.8787225676

0.8594314066

0.8388314735

0.8169541386

0.7938327175

0.7695024201

0.7440002975

0.7173651853

0.6896376443

0.6608598989

0.6310757730

0.6003306228

0.5686712681

0.5361459208

0.5028041118

0.4686966151
51630629880 98237688900 65688277892 99755531027 38629070418 85727793386 43799251452 43634905493 09872674752 72445795953 71757654165 38770683194 78213828704 63111096977 80255275617 81463470371 35041373866 83597272317 62099880254 42027600771 86119801736 46871966248 29751743155 22709784725 97131932020 88784987594 70544477036 04605449949 0.0086839452
0.0017832807

0.0041470332 0.0065044579 0.0088467598 0.0111681394 0.0134630478 0.0157260304 0.0179517157 0.0201348231 0.0222701738 0.0243527025 0.0263774697 0.0283396726 0.0302346570 0.0320579283 0.0338051618 0.0354722132 0.0370551285 0.0385501531 0.0399537411 0.0412625632 0.0424735151 0.0435837245 0.0445905581 0.0454916279 0.0462847965 0.0469681828 0.0475401657 0.0479993885 0.0483447622 0.0485754674 0.0486909570

2169643294 6056246763 6897836285 2636394772 6013112881 9671864259 7602471932 7569734308 5353020937 0838325415 6871087333 1505465867 1425948322 7240247886 5485155358 3714160939 5688238381 4024004604 7861562912 3272034138 4262352861 2365358900 2932345337 6375656306 2741814448 8131441729 1621001732 1483030866 9645830772 3480295717 4150342693 0913972038

0.0011449500 0.0026635335 0.0041803131 0.0056909224 0.0071929047

0.0101617660

0.0116241141

0.0130687615

0.0144935080

0.0158961835

0.0172746520

0.0186268142

0.0199506108

0.0212440261

0.0225050902

0.0237318828

0.0249225357

0.0260752357

0.0271882275

0.0282598160

0.0292883695

0.0302723217

0.0312101741

0.0321004986

0.0329419393

0.0337332149

0.0344731204
0318694153 8951268166 2469489523 5140319864 6926085842 4110306452 2079782691 9240133929 4050907611 8372568804 5626930635 0829903142 7814199892 1578200638 4633246192 6593010129 6411549110 6756511790 0048638067 5727686239 8326784769 5955798066 8811470164 7348777314 9764540138 8461152281 5175392879 6811731275

$0.4338753708 \quad .31756093062$ $0.3983934058 \quad 81969227024$ $0.3623047534 \quad 99487315619$ $0.3256643707 \quad 47701914619$ $0.2885280548 \quad 84511853109$ $\begin{array}{lll}0.2509523583 & 92272120493\end{array}$ $0.2129945028 \quad 57666132572$ $0.1747122918 \quad 32646812559$ $0.1361640228 \quad 09143886559$ $0.0974083984 \quad 41584599063$ $0.0585044371 \quad 52420668629$ 0.019511383256793997654

$\mathrm{n}=96$

$0.9996895038 \quad 83230766828$ $0.9983643758 \quad 63181677724$ $0.9959818429 \quad 87209290650$ $0.9925439003 \quad 23762624572$ $0.9880541263-29623799481$ $0.9825172635 \quad 63014677447$ $0.9759391745 \quad 85136466453$

$0.9683268284 \quad 63264212174$ $0.9596882914 \quad 48742539300$ $0.9500327177 \quad 84437635756$ $0.9393703397 \quad 52755216932$ $0.9277124567 \quad 22308690965$ 0.915071423120898074206 $0.9014606353 \quad 15852341319$ $0.8868945174 \quad 02420416057$ $0.8713885059 \quad 09296502874$ $0.8549590334 \quad 34601455463$ $0.8376235112 \quad 28187121494$ $0.8194003107 \quad 37931675539$ $0.8003087441 \quad 39140817229$ $0.7803690438 \quad 67433217604$ $0.7596023411 \quad 76647498703$ $0.7380306437 \quad 44400132851$ $0.7156768123 \quad 48967626225$ $0.6925645366 \quad 42171561344$ 0.668718310043916153953 $0.6441634037 \quad 84967106798$ 0.618925840125468570386 